\title{
Avoiding the Mishaps of Healthcare Provision
}

\author{
Geneva M Edwards*
}

Medical Investigator, Unlawful Medicine LLC, Buford, Georgia

*Corresponding author: Geneva E, MD, Medical Investigator, Unlawful Medicine LLC, Buford, Georgia, Tel: 678-471-6863, Fax: 678-828-5990, E-mail: drgedwards@aol.com

Citation: Geneva E (2018) Avoiding the Mishaps of Healthcare Provision. J Nurs Healthcare Manage 1: 201

Article history: Received: 27 July 2018, Accepted: 15 October 2018, Published: 17 October 2018

\begin{abstract}
Legal Medicine consists of a broad range of legal, medical, and ethical issues, as well as human rights and rights of individuals. This discipline specialty is necessary in the healthcare profession because healthcare providers have a duty to act in a patients' best interest always. If healthcare professions fail to meet this duty they can and will be charged in a court of law. Medical Malpractice is defined as the legal cause of action that occurs when a healthcare professional deviates from the standard of care in his or her profession, thereby causing harm to a patient and happens when a patient is harmed by a healthcare professional who failed to meet the standard of care. When a healthcare provider does not meet the standard of care, they have breached their contract with their patient.

The concept of the standard of care is often discussed among healthcare providers, and yet the legal definition of this term is frequently not understood. It is estimated that 7-17 malpractice claims are filed per 100 healthcare providers every year with emergency health care providers on the front lines in healthcare provision and therefore are frequently involved in medical malpractice cases. Many healthcare professionals need to review evidence-based practices that focus on standard of care and review the best research and clinical expertise to assist them in meeting the needs of patients, far beyond optimum care provision; helping them avoid any mishaps along the way.
\end{abstract}

Keywords: Healthcare Provision; Legal Medicine; Medical Malpractice; Provider-Patient Relationship

\section{Introduction}

Over the years, the healthcare profession has evolved into a business, an organization that is plagued with an epidemic, an ailment known as "Negligence". When you compare the healthcare profession yesterday to today, you will find similarities as well as differences. As far as difference today, our society is No stranger to malpractices lawsuits and have no problem focusing in on healthcare providers, holding them responsible and liable for all their actions.

It is worth noting that back in the fifties, healthcare providers; especially doctors were looked upon as Gods and could do no wrong. Even in the sixties and seventies, healthcare providers were highly respected. Today health care providers are still respected, but now things are quite different. For example, today, people are given access to tons of information about health care, provision of health care, and have social media, keeping them aware of their rights as a patient, etc. These factors may in part, contribute to malpractice claims. And there are lawyers who specialize in malpractice, invest a lot of funds in advertising their expertise, and influence patients and their families to focus on health care providers and possible wrong doings. Unfortunately, these tactics predispose healthcare providers to medical malpractice suits on, an ongoing basis [1]. However, the specialty lawyers are not the main problem when it comes to malpractice suits. The root of the problem in malpractice claims is the failure of a health care provider to meet the standard of care that results in harm to a patient.

\section{Standard of Care}

What is the standard of care about? The concept of the standard of care is often discussed among healthcare providers, and yet the legal definition of this term is frequently not understood. This theory is necessary for a review, as it serves as guidelines in care provision and is referenced, to show evidence that patients are provided the best of care in every situation. Since these are guiding principles and not God-lines, they are used for justice purposes only. When healthcare providers find themselves in legal trouble it usually falls within the area of not meeting the standard of care. Legally, the term standard of care refers to an ethical or legal duty of a professional to exercise the level of care, diligence, and skill prescribed in the code of practice of his or her profession, or as other professionals in the same discipline would in the same or similar circumstances. Failure to meet the standard of care is considered negligence. However, the healthcare provider will be held liable only if damages were caused by such negligence. The problem with the standard of care is that it is not subject to a specific definition and is judged on a case by case basis. The standards specifically 
for professionals are established by the practice of other professionals in their community of work. For example, in the healthcare profession there is a medical standard of care; defined as the level and type of care that a reasonably competent and skilled healthcare professional, one with a similar background who works in the same medical community and would have provided care under the same circumstances that led to the alleged malpractice claim. These providers are often called upon as "expert witnesses". [2].The purpose of expert witnesses is for both provider and patient. Because, a provider's lawyer wants to prove that the provider's actions were aligned with the standard of care, and the patient's lawyer wants to prove that the provider did not meet the standard of care and was therefore negligent. What constitutes the standard of care will change from community to community as well as evolve over time. But the standard of care in legal settings is not written down, it is provided by testimony by expert witnesses [3].

\section{Medical Ethics}

Deontology v. Medical Ethics. Deontology is a way people judge the morality of other based on a set of rules, maintains that actions are not justified by their consequences; rather, factors other than good outcomes determine the rightness of actions. Deontology theories necessarily generate that duties are independent of any theory of good, categorically imperatives. Ethics is the study of morality and how to consistently practice perfect character formation and behavior. Medical ethics is based on a set of values that professionals can refer to in the case of any confusion or conflict. A medical ethics committee focuses on medical issues and concerns, and consists of doctors, nurses, social workers, chaplains, and members of the community who are available to help patients, families, doctors, and other healthcare providers when they face difficult ethical decisions.

Healthcare providers have a duty to act in the patient's best interest always, refraining from exploiting the patient. This is a called a fiduciary relationship. Any behavior on the part of a healthcare provider that violates the limits of a professional relationship is called a boundary violation. In clinical practice, technical errors caused by wrong options are common, but on the other hand, ethical errors which are rare, are potentially more harmful to both patients and health care providers. For example, if a healthcare provider violates the boundary, it predisposes both the patient and the provider to harm.

Boundary violations are different from boundary crossings [4]. For example, if a provider develops a business relationship with a patient, this is neither harmful nor unethical, if the provider does not violate patient's confidentiality, or if a provider just happens by chance to see a patient in a social setting, these both are considered boundary crossings. On the other hand, if a provider developed a business relationship with the patient to take advantage of confidential information (personal or health) that the patient has shared with them, or if a provider planned to meet the patient in a social setting, these are unethical, inappropriate behaviors, and are boundary violations [5].

Exploitation examples include social or personal violations, business relationships and/or sexual relationships with patients [1]. It are the boundary violations that are unethical, cause the most harm to patient and the provider, and therefore are the major concern in provider-patient relationships.

\section{Examples of boundary violations (unethical behavior)}

1) An upsurge pattern of increasing intrusion into a patient's space that culminates in sexual contact

2) Engaging in a sexual relationship with a patient

3) A provider giving a patient gifts (monetary and other), to manipulate them to benefit them.

\section{Medical Malpractice}

A healthcare provider has a duty of care to patients; an established contract. The contract is not required to be in writing to be valid. There are two main types of contracts, expressed contracts and implied contracts. An expressed contract is clearly stated in written or spoken words. A payment contract is an example of an expressed contract. Implied contracts are those in which the acceptance or conduct of the parties, rather than expressed words, creates the contract. For example, a patient who stretches out their arm for a blood draw or for an injection is creating an implied contract, giving the healthcare professional consent to 93 proceed [6].

Healthcare providers are held to a higher standard than the general members of the public. This higher standard affects both their clinical and personal lifestyle when they are not at work. Therefore, it is their behavior and conduct that they are held most accountable to. When a healthcare provider does not meet the duty of care, it is called a breach of contract. There may be misconceptions that the only healthcare provider who has a contract with a patient is a physician. However, any healthcare provider who provides patient care and/or technician, nurse, pharmacist, or other healthcare professional [1].

According to the National Institute of Health, medical malpractice is defined as professional negligence by a healthcare provider who provides treatment below the standard of care, causing harm, injury or death to a patient. Emergency room providers are on the front lines of healthcare and are frequently involved in medical malpractice claims. It is estimated that 7-17 medical malpractice claims are filed per 100 healthcare providers every year [7].

Negligence is a common medical malpractice claim, involving inappropriate, unqualified, or sub-standard care provision of a patient by a physician, dentist, nurse, pharmacist, or other healthcare professional. Negligence is the chief model of liability concerning contentions of medical malpractice, making this type of lawsuit part of Tort Law. To successfully claim negligence, a patient must show tangible compensable injury caused by the negligent healthcare provider [8]. 


\section{Means of proof and testimony of medical malpractice}

There are 4 means of proof and the testimony to be brought to justice to support a malpractice claim: a professional duty owed to the patient, breach of duty, injury caused by the breach and resulting damages. All four must be proven for a successful malpractice lawsuit.

\section{Preventable hospital deaths}

More than 1000 people die daily, approximately 444,000 die yearly from preventable hospital errors. Medical errors now claim the third leading cause of death in the United States. Patients are not dying from the illnesses that caused them to seek hospital care in the first place. Patients are dying from mishaps that hospitals and/or healthcare professionals could have prevented [9].

\section{Medication errors}

According to the 2015 Forbes report, medication errors happen quite often, not to mention the medication errors that go unreported. An estimated 1 million medication errors occur each year, contributing to more than 7,000 deaths annually. On average, there is 1 medication error every day for every patient in an inpatient setting. Medication errors have been recently observed in almost 50\% of all surgical procedures with the most frequent observed mistakes in the labeling of medications, incorrect dosages, neglecting to treat a problem indicated by the patient's vital signs, as well as documentation errors. Medication errors among patients in outpatient settings are primary caused by transcription errors, either by the provider's handwritten prescription or by the pharmacist who misreads the provider's handwritten script when completing the prescribed medication order. Also, there are times when patients misread the label on the medication or decide to adjust the medication prescribed according to their own Understanding [10].

Let's take a closer look at how we can prevent medication errors. According to recent research, the best-known way for hospitals to protect patients from medication errors is by adopting a technology to prevent the misreading of prescriptions; a technology called computerized physician order entry (CPOE). This computer software allows a provider to enter medication orders for a patient on a computer that contains patient information such as key lab values, clinical condition, allergies, etc. Once the provider enters a medication order, the CPOE checks the safety and appropriateness of the order and sends it electronically to the pharmacy.

The CPOE system would also alert the provider to a misplaced decimal in a medication order. One of the greatest advantages of the CPOE system is that it abolishes the need for pharmacists to interpret a provider's handwriting [11].

\section{Errors arising from a provider's clinical documentation}

A very important fact learned in basic nursing school: "If it wasn't documented, it didn't happen". Many malpractice suits arise from inadequate documentation, erroneous documentation, and/or no documentation at all.

\section{Failure to document significant changes}

Many medical errors arise from clinical documentation issues, such as failure to document, evidence of infection, wound appearance, wound size, drainage and/or odor present in wound, signs of dehydration such as increased weakness, fatigue, and tingling pain in hands, etc., and failure to document the initiation of emergent care for falls w/injury or other changes noted in the patient's status from a fall [12].

\section{When healthcare provision and the law collide}

The most common medical malpractice claims in the United States are, Misdiagnosis and Failure to Diagnose, Prescription Errors, Childbirth Injuries, Surgical Errors, and Anesthesia Errors [6]. The following are court case examples of the most common medical malpractice claims:

\section{Misdiagnosis and Delayed Diagnosis: Jane Doe v. State of New York}

A forty-year-old woman in New York felt a lump in her breast during a self-exam, went to her doctor, and was referred to have a mammogram. After the mammogram, the radiologist treating her looked at the scans, and believed that her lump was a clogged milk duct. However, the lump did not go away, continued to grow over the next year so she went to the doctor again and was diagnosed with cancer and because the cancer had not been treated, it had spread to her lymph nodes. She had to undergo a mastectomy, radiation and chemotherapy. Because of the cancer being advanced in her breast it spread to her bloodstream, and her bones. During the trial, the courts looked at the fact that her metastatic breast cancer would be why she would not live longer. The radiology's negligence resulted in severe emotional trauma, pending premature death and as a result, she was awarded \$15 million dollars in damages [13].

\section{Prescription Error: Doe v Magsaysay, Cook County Illinois}

A doctor gave a patient the wrong prescription. As a result, the patient began to developed pancreatitis and needed a blood transfusion for treatment. During the transfusion, the patient became infected with HIV. He sued his doctor for malpractice because of the original prescription error and the jury awarded him $\$ 2,400,000 ; \$ 1,000,000$ for loss of services and $\$ 1,400,000$ for punitive damages [14]. 


\section{Childbirth Injuries: 1-McQuitty v. Dr. Spangler, 2009: Birth Injury/Informed Consent}

In 2009, the court found that an informed consent lawsuit in Maryland can stand where the healthcare provider withholds material information about a proposed course of medical treatment, or about an ongoing course of medical treatment. The patient filed a negligence; alleging that the healthcare provider breached the duty to obtain her informed consent. The allegation is that when the healthcare provider failed to inform the mother, who was hospitalized for a partial-placental-abruption, of risks and available alternative treatments related to material changes in her pregnancy: a second partial-placental-abruption, oligohydramnios, and intrauterine growth restriction. McQuitty's baby was born with severe Cerebral Palsy and the Maryland court awarded the family over \$13 million dollars [15].

\section{Childbirth Injuries: 2-Doe v. State of California 2018}

In 2016, Negligence and Falsification of Records was number 1, of ten top malpractice claims in the United States, against a California health care provider who had been negligent in the delivery of a baby. The provider did not perform an episiotomy, did not use forceps during the delivery, failed to assess contractions, and unfortunately, the baby was born with Cerebral Palsy. The hospital refused to accept the provider was negligent, so they chose to falsify their records; resulting in a \$75 million-dollar settlement claim, for the defendants. This is a good example of a hospital covering up the wrong doings, causing more liabilities [16].

\section{Surgical Error: Gerato v. University of Florida Shands Hospital 2006}

The surgical team of Shands Hospital in Florida unknowingly perforated one of the arteries in her brain during a procedure. From that point on errors continued, leading to the tragic outcome of her becoming paralyzed. The court held the entire surgical team liable and awarded Gerato $\$ 23$ million dollars in this medical malpractice suit [17].

\section{Anesthesia Error: Anesthesiology Malpractice Settlement for Brain Damaged Woman}

A 49-year old woman who presented to the hospital for elective colon surgery. Prior to going to the operating room, a thoracic epidural was placed for post-operative pain control. Before surgery even began, the anesthesia assistant activated the epidural without approval or direction of the anesthesiologist. The defendant anesthesiologist then entered the room where an IV anesthesia was given to the patient; Lidocaine, Fentanyl, Propofol, and Nimbex. The main anesthesiology left the room, leaving the anesthesiology assistant in charge. The patient's heart rate and blood pressure dropped immediately. The assistant anesthesiologist failed to act promptly, and because of the anesthesiologist's negligence, the patient suffered a significant hypoxic injury to her brain that left her permanently disabled [18].

\section{Steps to avoid the mishaps of healthcare provision}

Listed above are the most common examples of case studies involving medical malpractice suits but there are many more that affect healthcare provision. For example, mishaps such as: clinical documentation errors, unauthorized practice of medicine, wrongful deaths, etc. When caring for a patient, a provider's priority is to maintain a patient's safety at all time. Safety includes a wide range of techniques; including providing a safe environment for patients in areas, providers have control of, and troubleshooting situations that may be harmful to their patients so those situations can be prevented. It's worth noting that all mishaps of healthcare provision are centered around safe clinical practices.

If a healthcare provider has questions about a specific care provision, they should reach out to other healthcare providers who may have more experience in the area and/or healthcare providers who are a subject matter expert (SME) in that specialty. A SME may be aware of an updated policies and procedures in the area that the healthcare provider does not know about. Or the SME may be able to bring attention to common errors of omission, which are common in caring for patients and could threaten the safety of a patient. Safety and policy change go hand in hand when it comes to providing patients safe, quality care. Also, providers need to consult SMES to look at workloads, to make sure that a healthcare provider is not placed in a situation that will alter the safety of the patients being cared for. Whether there is a question on pressure ulcers, suicide prevention, drug therapy, allergic reactions, and workloads. etc., healthcare providers should not hesitate to ask as expert in those areas of expertise.

\section{Common prevention tactics to avoid mishaps of healthcare provision}

\section{Safe Medication Administration to Prevent Medication Errors}

If the 5 R's (Right Patient, Right Dose, Right Drug, Right Route and Right Time) were always followed when overseeing medications there would certainly be less medication administration errors [19]. There are some healthcare facilities that have a safety medication team, initiated by the pharmacy department to guide in the clinical practice of safe medication administrations. If a provider works for a healthcare facility that does not have a safety medication team, this is an opportunity for a provider to initiate and implement one to maintain medication safety in every way possible. 


\section{Proper Clinical Documentation}

As stated before, "if it wasn't documented it wasn't done." Many malpractice suits of negligence have been successful because of improper documentation which includes poor or no documentation at all. Clinical documentation in a patient's record should read like a book so anyone who reads it will clearly understand what's going on with the patient and the treatment plan that is followed. Risk manager's programs in clinical settings emphasize the importance of proper documentation and strive for healthcare providers to document detailed facts of a patient's care, in a timely manner [20]. Many risk management programs are staffed with at least one legal nurse consultant, who should be consulted whenever a question rises on proper documentation. This could save the provider as well as the healthcare facility from legal implications.

\section{Initiating and Maintaining Fall-Prevention Programs}

To prevent falls and injuries to patients, maintaining a safe milieu is a must when patients are receiving care. Approximately 700,000 to 1,000,000 patients experience falls in United States Hospitals annually, 30 to 50\% of those falls result in serious injuries and fatalities.

According to the 2016 2nd quarter sentinel report of the Joint Commission, formerly known as the Joint Commission on Accreditation of Healthcare Organizations (JCAHO), the root cause analysis of hospital falls shows that there are many factors that contribute to patient falls, which include the following: Poor physical assessment of patients by healthcare providers, insufficient communication among healthcare staff, hospital providers and staff not following safety practices, provider-patient overload in patient care assignments, and lack of orientation provided to patients of their new environment when they are admitted to the hospital. All patient falls are not avoidable, but according to research published by the National Institute of Health (NIH), $1 / 3$ of all hospital falls are preventable. These findings make it imperative for hospitals to construct and maintain a fall prevention environment, one that trains staff, and prompts patients and family members to avert falls. Atmospheres that encourage the use of standardized fall risk assessment tools, as well as promote methods the hospitals should successfully practice, will decrease the number of patient falls within their healthcare organizations [21].

\section{Preventing Pressure Ulcers}

Maintaining patient safety covers all areas in healthcare provision, including prevention and care of pressure ulcers in patients. Preventing pressure ulcers has been a great concern of nursing care for many years. Pressure ulcers are more common in the elderly population because of compromised immune systems which comes with aging, acquired morbidities and co-morbidities. Pressure ulcers are also more common in nursing homes because the patients are kept sedentary and positions maintained in bed and/or wheelchairs. The development of pressure ulcers may be in inevitable in some patients but the most important technique a provider can initiate is a prevention treatment plan, one that promotes frequent turning, protein-rich nutritional supplements to promote healing, and proper clinical documentation of wound progression (size of wound, area involved, drainage, odor, surface Area, etc.). Clinical documentation from a wound specialist should also be available in the patient's chart to exhibit that the wound care is guided in perfection [22].

The prevention of falls and pressure ulcers are relevant issues for quality improvement of hospitals and other healthcare facilities. However, failure to identify a fall-risk patient in which can prevent a fall, and/or overlooking a patient who is vulnerable for developing pressure ulcers, or improperly documenting stages of pressure ulcers, are all calamities in the making. Providers should consult with wound specialists' practitioners, to guide their practice in Fall prevention and prevention and care of pressurulcers.

\section{Conclusion}

I don't believe that there is one healthcare provider who intentionally places a patient in danger or has a desire to harm or cause injury to a patient. However, healthcare providers are pressured in many ways that often produce an unsafe environment for their patients. For example, time is often a factor and providers many times find themselves caring for more patients than they have time to properly care for. Hospital employers do not always support Healthcare providers working beyond their tour of duty and these results in rushing to get the job done, including proper clinical documentation, within assigned work hours. Some healthcare providers work long hours and work overtime as needed. Research has proven that heavy workloads, because of inadequate staffing, result in nurses providing care beyond the point of effectiveness. In fact, according to the Institute of Medicine, more medical errors are made when providers work long hours and excessive overtime [23]. Carelessness is a by-product produced of providers who do not take the time or have enough time to perform their necessary duties in healthcare provision. There are two sure ways providers can be successful in avoiding the mishaps of healthcare provision. One way is to communicate effectively. Patients are less likely to sue if their provider has gained confidence with pleasant, direct communication, explaining in detail pending procedures, etc.), another way a provider can prevent the mishaps of healthcare provision is to practice safe-care measures combined with providing quality care, always. Avoiding mishaps of healthcare provision may be challenging but, regardless of how difficult this may be at times, these are the only definite ways a healthcare provider will protect themselves from malpractice suits [23,24]. 


\section{References}

1. Santucci NM (2014) Legal Aspects of Health Care Administration. Sudbury: Jones \& Bartlett Learning.

2. 1.04, C. (n.d.), Understand legal and ethical issues in healthcare. Retrieved from Contracts.

3. Poghosyan L, Norful AA, Fleck E, Bruzzese JM, Talsma A, et al. (2017) Primary Care Providers' Perspectives on Errors of Omission. J Am Board Fam Med 30: $733-42$.

4. Strong J (2013) McCormick on Evidence (4th Edn). St Paul: West Publishing Company.

5. Moore JP, Adler NE, Robertson PA (2000) US National Library of Medicine. Medical Malpractice.

6. Knickman RJ, Anthony RK (2014) Health Care Delivery in the United States, $11^{\text {th }}$ Edn. New York.

7. Kessler DP (2011). Evaluating the Medical Malpractice System and Options for Reform . J Econ Perspect, US Library of National Science. 25: $93-110$.

8. Adriel B, David CR (2011) The Integration of Emotions in Memories: Cognitive-Emotional Distinctiveness and Posttraumatic Stress Disorder. Appl Cogn Psychol US Library of Science 25: 811-6.

9. Shanafelt TS, Sinsky CA, Stephen S (2017) Preventable Deaths in American Hospitals NEJM Catalyst. Retrieved from Preventable Deaths in American Hospitals. 10. Binder L (2013). NEJM Catalyst, Forbes Pharma and Healthcare. Retrieved from The Shocking Truth About Medication Errors.

11. Computer Physician Order Entry, CPOE (2017) Retrieved from Patient Safety Primer.

12. Funicari M (2016) The 10 Most Common EHR Documentation Errors. Retrieved from Chiro Health USA.

13. Fisher $J$ (2018) Protecting patient rights blog.

14. Rosenfield J (2018) Rosenfield Injury Lawyers LLC.

15. FindLaw (2009) McQuitty v. Spangler.

16. Liebeck HM (2018) Verdict victory blog.

17. Grossman (2006) Jury Awards Florida Woman \$23 Million in Medical Malpractice Lawsuit. Retrieved from GYRC Trial Lawyers.

18. Schwartz S (2017) \$3,045,000 Anesthesiology Malpractice Settlement for Brain Damaged Woman Sommers Schwartz PC verdict.

19. Institute for Safe Medication Practices (2017) ISMP Medication Safety Alert.

20. Natale C (2012) Why clinical documentation improvement is so important to hospitals.

21. Huges R (2008) Fall and Injury Prevention, Chapter 10. Retrieved from Agency for Healthcare Research and Quality US.

22. Huges R (2008) Pressure Ulcers: A Patient Safety Issue.

23. Harris CR, Warren R, Jack EF (2006) Professional Negligence: When Practice Goes Wrong. The Annals of Pharmacotherapy, 40: 1377-82.

24. WFTV9 (2013) Retrieved from Central Florida doctor fired from job, accused of sexualmisconduct. 